\title{
Three New Mersenne Primes and a Statistical Theory
}

\author{
By Donald B. Gillies
}

If $p$ is prime, $M_{p}=2^{p}-1$ is called a Mersenne number. If $u_{1}=4$ and $u_{i+1}=$ $u_{i+1}^{2}-2$, then $M_{p}$ is prime if and only if $u_{p-1} \equiv 0\left(\bmod M_{p}\right)$. This is called the Lucas test (see Lehmer [4]).

The primes $M_{9689}, M_{9941}$, and $M_{11213}$ which are now the largest known primes, were discovered by Illiac II at the Digital Computer Laboratory of the University of Illinois. The computing times were 1 hour 23 minutes, 1 hour 30 minutes, and 2 hours 15 minutes respectively, and the calculations were checked by repetition. This brings to 23 the number of known Mersenne primes, namely for

$$
\begin{gathered}
p=2,3,5,7,13,17,19,31,61,89,107,127,521,607,1279,2203,2281,3217, \\
4253,4423,9689,9941,11213 .
\end{gathered}
$$

The Lucas test was applied to all $M_{p}, p<12,000$ for which no factor is known and the residue $u_{p-1}$ was printed in modified octal (one base 4 digit and 14 octal digits per word). It was verified that there are just 20 prime $M_{p}$ for $p<7000$. The last five octal digits of the residues, $R$, are shown in Table 3 for $7000<p<$ 12,000. As an indication of the speed of Illiac II, the residue for $M_{8191}$ took 100 hours on Illiac I (D. J. Wheeler), 5.2 hours on an IBM 7090 (Hurwitz [2]) and 49 minutes on Illiac II. The three values agree.

An Illiac II word has a 45-bit mantissa and a 7-bit exponent. The program checks the multiple precision arithmetic modulo $2^{44}-1$. Four errors were found in the residues given in Hurwitz [2], namely

\begin{tabular}{c|c|c}
\hline$p$ & Correct Residue & Hurwitz' Residue \\
\hline 3637 & 53313 & 67413 \\
3847 & 14400 & 57652 \\
4397 & 44327 & 40174 \\
4421 & 03013 & 25131 \\
\hline
\end{tabular}

and ten errors were found in the results of M. Berg and S. Kravitz, privately communicated, for the range $6000<p<7000$. For the range $5000<p<6000$ the residues agree with those obtained by Hurwitz and Selfridge. In no case has the Illiac II program obtained different results on different runs.

The author is indebted to $\mathrm{J}$. Brillhart for a list of prime factors $<2^{34}$ of Mersenne numbers. For the range $5000<p<17,000$ a wider range factor table was computed by Illiac II and Table 4 gives factors $q$ in the range $2^{34} \leqq$ prime factor $\leqq$ $2^{36}$ not covered by Brillhart. These were used to exclude some values $M_{p}$ from the Lucas test.

Received June 1, 1963. Revised August 23, 1963. 
For $p=5387,5591,5641,5987,6089,6661,6779,6907$ and certain larger values, Table 4 gives the first known prime factor. The factor program was written after the Lucas residues were communicated to Kravitz and Berg.

The very large gap in $p, 4423$ to 9689 , between successive Mersenne primes raises the question of what the distribution is of Mersenne primes and of factors of Mersenne composites. I. J. Good [1] suggested that the number of prime $M_{p}<x$ is asymptotic to $2.3 \log \log x$, and $\mathrm{D}$. Shanks [7] in effect suggests approximately $\frac{5}{\log 10} \log \log x$. It will be suggested in what follows that $\frac{2}{\log 2} \log \log x$ is the correct asymptotic function. Since the number of Mersenne primes is a slowly varying function of $x$, a stronger assumption will be made, which implies the above and can be tested by studying the distribution of prime factors of Mersenne composites.

It has been proved by Fermat and Euler that all factors of $M_{p}$ must be of the form $2 k p+1$ and simultaneously of the form $8 l \pm 1$. This means that, depending on $p, k$ is constrained to one of the sequences

$$
1,4,5,8,9,12, \cdots \text { and } 3,4,7,8,11,12, \cdots \text {. }
$$

Thus the smallest potential factor is either $2 p+1$ or $6 p+1$ and thereafter potential factors are spaced an average of $4 p$ apart. In the conjecture that follows, we suggest that statistically, this theorem sets a lower bound on potential divisors, but does not change the expected probability density of divisors above that lower bound-the low density of potential divisors is just compensated for by a high conditional probability that any one of these actually divides $M_{p}$.

The prime number theorem implies that a randomly chosen integer in the range $s$ to $s+\Delta s$ is prime with probability $\sim \frac{1}{\log s}$, as $s, \Delta s \rightarrow \infty$ but $\frac{\Delta s}{s} \rightarrow 0$, and that the probability that such a number is prime and also divides some large random number $N \gg s$ is $\frac{1}{s \log s}$.

As $A, B-A, N \rightarrow \infty$, the expected number of prime divisors of $N$ in the range $A \leqq$ prime divisor $\leqq B \leqq \sqrt{N}$ is

$$
\sim \int_{A}^{B} \frac{d s}{s \log s}=\log (\log B / \log A) \text {. }
$$

Conjecture. If $A<B \leqq \sqrt{M}_{p}$, as $B / A$ and $M_{p} \rightarrow \infty$, the number of prime divisors of $M_{p}$ in the interval $[A, B]$ is Poisson distributed with

$$
\begin{aligned}
\text { mean } & \sim \log (\log B / \log A) \text { if } A \geqq 2 p \\
\quad \text { or } & \sim \log (\log B / \log 2 p) \text { if } A<2 p .
\end{aligned}
$$

If true, this conjecture implies that

(1) The number of Mersenne primes less than $x$ is $\sim \frac{2}{\log 2} \log \log x$.

(2) The expected number of Mersenne primes in the interval $[x, 2 x]$ in $p$ is $2+2 \log \left(\frac{\log 2 x}{\log x}\right)$ which is $\sim 2$.

(3) The probability that $M_{p}$ is prime is $\sim \frac{2 \log 2 p}{p \log 2}$. 
The observed frequency of Mersenne primes in the interval $[x, 2 x]$ in $p$ is shown in

TABLE 1

\begin{tabular}{c|c|c|c|c|c|c|c|c|c|c|c}
\hline$x$ & 5 & 10 & 20 & 40 & 80 & 160 & 320 & 640 & 1280 & 2560 & 5120 \\
\hline $\begin{array}{c}\text { Number of primes } \\
\text { in }[x, 2 x]\end{array}$ & 2 & 3 & 1 & 1 & 3 & 0 & 2 & 1 & 2 & 3 & 2 \\
\hline
\end{tabular}

The total number of prime factors less than $2^{36}$ of Mersenne numbers in the interval $5000<p<7000$ was estimated as

$$
[\pi(7000)-\pi(5000)] \log \left(\frac{\log 2^{36}}{\log 12,000}\right)
$$

and was also counted using the factor table prepared. Similar calculations were done for other intervals of length 2000 in $p$ and the results are shown in

TABLE 2

\begin{tabular}{c|c|c|c|c|c|c}
\hline Range of $p$ & $\begin{array}{c}5000 \text { to } \\
7000\end{array}$ & $\begin{array}{c}7000 \text { to } \\
9000\end{array}$ & $\begin{array}{c}9000 \text { to } \\
11000\end{array}$ & $\begin{array}{c}11000 \text { to } \\
13000\end{array}$ & $\begin{array}{c}13000 \text { to } \\
15000\end{array}$ & $\begin{array}{c}15000 \text { to } \\
17000\end{array}$ \\
\hline $\begin{array}{c}\text { Prime Factors }<2^{36} \\
\text { observed }\end{array}$ & 223 & 209 & 206 & 192 & 175 & 169 \\
\hline Predicted number & 226 & 205 & 206 & 192 & 184 & 181 \\
\hline
\end{tabular}

Digital Computer Laboratory

University of Illinois

Urbana, Illinois

1. I. J. Good, "Conjectures concerning Mersenne numbers," $M T A C$ v. 9, 1955, p. 120, 121.

2. A. Horwitz, "New Mersenne primes," Math. Comp. v. 16, 1962, p. 249-251.

3. S. Kravitz, "Divisors of Mersenne numbers $10,000<p<15,000$," Math. Comp. v. 15,1961, p. 292-293.

4. D. H. LeHMER, "An extended theory of Lucas' functions," Ann. of Math. v. 31, 1930, p. $419-448$.

5. H. Riesel, "Mersenne numbers," MTAC v. 12, 1958, p. 207-213.

6. H. RisseL, "All factors $q<10^{8}$ in all Mersenne numbers $2^{p}-1, p$ prime $<10^{4}$," Math. Comp. v. 16, 1962, p. 478-482.

7. D. SHANKs, Solved and Unsolved Problems in Number Theory, Spartan Books, Washington, 1962 , p. 198 . 
TABLE 3. Lucas Residues $R$ for $7000<p<12,000$

\begin{tabular}{|c|c|c|c|c|c|}
\hline$p$ & $R$ & $p$ & $R$ & $p$ & $R$ \\
\hline 7069 & 07313 & 8887 & 23230 & 10501 & 77772 \\
\hline 7109 & 76754 & 8893 & 47223 & 10531 & 35724 \\
\hline 7121 & 11254 & 8923 & 41014 & 10597 & 42547 \\
\hline 7127 & 70746 & 8941 & 01034 & 10639 & 70051 \\
\hline 7177 & 64624 & 8999 & 36025 & 10667 & 55573 \\
\hline 7213 & 35572 & 9011 & 74242 & 10709 & 21742 \\
\hline 7229 & 21270 & 9013 & 56660 & 10711 & 33125 \\
\hline 7237 & 44431 & 9041 & 56532 & 10723 & 26405 \\
\hline 7243 & 75126 & 9091 & 53605 & 10729 & 55703 \\
\hline 7247 & 21344 & 9133 & 17234 & 10771 & 50555 \\
\hline 7309 & 16030 & 9151 & 36255 & 10781 & 00164 \\
\hline 7321 & 03466 & 9187 & 75427 & 10789 & 27651 \\
\hline 7331 & 55736 & 9203 & 23113 & 10831 & 42672 \\
\hline 7333 & 40632 & 9209 & 75536 & 10909 & 63265 \\
\hline 7351 & 43525 & 9227 & 07577 & 10937 & 47503 \\
\hline 7369 & 60027 & 9241 & 25121 & 10939 & 63332 \\
\hline 7433 & 13456 & 9257 & 62553 & 10957 & 64103 \\
\hline 7477 & 34613 & 9277 & 02036 & 11003 & 37656 \\
\hline 7481 & 56621 & 9281 & 62622 & 11027 & 10024 \\
\hline 7489 & 31253 & 9319 & 62503 & 11057 & 60335 \\
\hline 7507 & 62414 & 9377 & 66277 & 11069 & 64651 \\
\hline 7523 & 54030 & 9413 & 17541 & 11093 & 03231 \\
\hline 7559 & 01556 & 9433 & 25035 & 11113 & 24506 \\
\hline 7577 & 74000 & 9437 & 43446 & 11117 & 57045 \\
\hline 7603 & 42610 & 9463 & 63616 & 11131 & 50752 \\
\hline 7607 & 04024 & 9473 & 02247 & 11159 & 12377 \\
\hline 7621 & 43263 & 9533 & 43134 & 11161 & 50166 \\
\hline 7649 & 17277 & 9551 & 33274 & 11177 & 45677 \\
\hline 7699 & 15637 & 9587 & 05303 & 11213 & - \\
\hline 7703 & 11617 & 9623 & 26262 & 11239 & 44172 \\
\hline 7723 & 56523 & 9631 & 02445 & 11251 & 02106 \\
\hline 7727 & 65716 & 9649 & 10710 & 11257 & 17745 \\
\hline 7753 & 00553 & 9661 & 27003 & 11261 & 21260 \\
\hline 7757 & 35327 & 9679 & 75063 & 11279 & 40455 \\
\hline 7793 & 01165 & 9689 & - & 11351 & 22147 \\
\hline 7817 & 00650 & 9697 & 24211 & 11369 & 27670 \\
\hline 7867 & 12212 & 9719 & 32015 & 11383 & 17361 \\
\hline 7927 & 35532 & 9721 & 13527 & 11393 & 01511 \\
\hline 7937 & 36343 & 9749 & 37416 & 11411 & 74045 \\
\hline 7949 & 55402 & 9767 & 03263 & 11423 & 03630 \\
\hline 8009 & 55062 & 9769 & 51126 & 11443 & 65257 \\
\hline 8069 & 61334 & 9781 & 34012 & 11447 & 51354 \\
\hline 8089 & 24237 & 9787 & 21740 & 11467 & 55443 \\
\hline 8117 & 07547 & 9839 & 12364 & 11483 & 03450 \\
\hline 8147 & 32143 & 9857 & 23533 & 11489 & 57555 \\
\hline 8191 & 03624 & 9871 & 32316 & 11549 & 76107 \\
\hline 8233 & 75260 & 9887 & 33416 & 11551 & 47006 \\
\hline 8263 & 73755 & 9901 & 15526 & 11593 & 12504 \\
\hline 8291 & 31062 & 9907 & 01401 & 11597 & 07213 \\
\hline 8297 & 63521 & 9929 & 44612 & 11657 & 53703 \\
\hline 8311 & 72342 & 9931 & 25643 & 11681 & 13417 \\
\hline 8329 & 64615 & 9941 & - & 11689 & 23607 \\
\hline 8363 & 55516 & 9967 & 56346 & 11717 & 06015 \\
\hline 8369 & 76051 & 10037 & 34070 & 11743 & 76351 \\
\hline 8389 & 21672 & 10061 & 30610 & 11779 & 14053 \\
\hline 8443 & 14744 & 10069 & 23577 & 11789 & 71203 \\
\hline 8447 & 05235 & 10079 & 70743 & 11801 & 41730 \\
\hline 8521 & 47055 & 10099 & 11311 & 11807 & 64000 \\
\hline 8527 & 06705 & 10159 & 47661 & 11821 & 64453 \\
\hline 8543 & 63267 & 10169 & 37134 & 11839 & 25215 \\
\hline 8581 & 17603 & 10177 & 75465 & 11867 & 70645 \\
\hline 8609 & 52627 & 10259 & 72321 & 11887 & 10035 \\
\hline 8647 & 12277 & 10273 & 50763 & 11897 & 42220 \\
\hline 8681 & 35061 & 10303 & 21613 & 11927 & 14155 \\
\hline 8707 & 37065 & 10313 & 45100 & 11933 & 25571 \\
\hline 8753 & 32153 & 10343 & 40266 & 11941 & 23743 \\
\hline 8819 & $\begin{array}{l}32153 \\
24665\end{array}$ & 10369 & 21740 & 11987 & 33200 \\
\hline 8831 & $\begin{array}{l}24665 \\
51436\end{array}$ & 10399 & 12436 & 12043 & 05651 \\
\hline 8837 & $\begin{array}{l}51436 \\
25460\end{array}$ & 10453 & 62061 & 12071 & 40741 \\
\hline 8861 & $\begin{array}{l}20400 \\
22326\end{array}$ & 10463 & 52513 & 12097 & 51143 \\
\hline 8863 & 03214 & $\begin{array}{l}10477 \\
10487\end{array}$ & 67452 & 12109 & 20110 \\
\hline
\end{tabular}


TABLE 4

Prime Factors $q<2^{36}$ and $>2^{34}$ of $M_{p}$ for $5000<p<17,000$

\begin{tabular}{|c|c|c|c|}
\hline$p$ & $q$ & $p$ & $q$ \\
\hline 5297 & 45566266567 & 10631 & 31946920433 \\
\hline 5387 & 35028913631 & 10657 & 39041962129 \\
\hline 5591 & 52462455217 & 10883 & 22123941871 \\
\hline 5641 & 49748069257 & 11087 & 21232248047 \\
\hline 5987 & 60583758167 & 11197 & 33954656167 \\
\hline 6089 & 52969672361 & 11353 & 21935540009 \\
\hline 6427 & 52235468209 & 11399 & 21834921289 \\
\hline 6563 & 34128597577 & 11471 & 48126649327 \\
\hline 6607 & 26285724863 & 11617 & 56778668351 \\
\hline 6661 & 33930121529 & 11731 & 33949138609 \\
\hline 6673 & 19551502967 & 11827 & 41696632543 \\
\hline 6779 & 35331931073 & 11831 & 24583043351 \\
\hline 6907 & 60312932423 & 11863 & 17998472423 \\
\hline 6997 & 67983747617 & 11923 & 54654960463 \\
\hline 7001 & 39107810033 & 11953 & 26739410839 \\
\hline 7013 & 54547899457 & 11971 & 45647912969 \\
\hline 7307 & 42715085233 & 12373 & 23482147543 \\
\hline 7517 & 27743142241 & 12379 & 44996897503 \\
\hline 7669 & 40807239817 & 12437 & 35512186943 \\
\hline 7691 & 23104702303 & 12511 & 30515054639 \\
\hline 7717 & 57756173327 & 12577 & 25131864481 \\
\hline 7829 & 39555975527 & 12619 & 54388520951 \\
\hline 7933 & 46405939823 & 12641 & 18198590369 . \\
\hline \multirow[t]{2}{*}{8081} & 36263568311 . & & 41249479151 \\
\hline & 56994371767 & 12653 & 34300536887 \\
\hline 8093 & 29811811823 & 12659 & 47013095473 \\
\hline 8111 & 25919771153 & 12809 & 39970407647 \\
\hline 8573 & 21973130527 & 13001 & 18880338223 \\
\hline 8641 & 46880604889 & 13381 & 49300715543 \\
\hline 8747 & 21021997487 & 13451 & 25744353137 \\
\hline 8779 & 57336421553 & 13901 & 39030421543 \\
\hline 8783 & 46912673033 & 14011 & 52635964361 \\
\hline 8971 & 59568301217 & 14029 & 19858217849 \\
\hline 9043 & 37268518009 & 14389 & 52946109737 \\
\hline 9067 & 21497004703 & 14533 & 45439197527 \\
\hline 9619 & 53840698033 & 14593 & 63369110177 \\
\hline 9629 & 27152451199 & 14669 & 22222684199 \\
\hline 9803 & 63388001753 & 14717 & 25671863857 \\
\hline 9817 & 26619050743 & 14827 & 56787558271 \\
\hline 9883 & 21258273703 & 15233 & 67894547311 \\
\hline 10039 & 45613381103 & 15439 & 18568948471 \\
\hline 10067 & 34439287537 & 15461 & 58311965473 \\
\hline 10151 & 62820641017 & 15473 & 67117263047 \\
\hline 10267 & 35491478951 & 15913 & 18951969263 . \\
\hline 10427 & 35037410167 & & 29723415311 \\
\hline 10457 & 31794194791 & 16567 & 68395501007 \\
\hline 10529 & 37429900087 & 16889 & 49565127863 \\
\hline
\end{tabular}

